\title{
Speaker Abstracts
}

\section{New avenues of OA \& osteoporosis management}

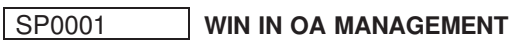

Francis Berenbaum. Sorbonne Universite, France

All recommendations on the management of osteoarthritis $(\mathrm{OA})$, including EULAR, consider that its treatment should include pharmacological and non-pharmacological treatments. In this lecture, the most recent advances in both fields will be addressed. Some of them can change our current practice like recommendations on physical activity or on the choice of analgesics.

Moreover, this lecture will highlight some of the drugs and cell therapies in development that could be on the market in the very close years

Disclosure of Interests None declared

DOI: 10.1136/annrheumdis-2019-eular.8428

\section{SP0002 HOT: OSTEOPOROSIS @2019}

Serge Ferrari. Geneva University Hospital, Medecine, Geneva, Switzerland

Background: The goal of osteoporosis therapy is to quickly prevent fragility fractures in subjects at risk and on the longer term to restore bone mass (Bone mineral density, BMD) to levels of appropriate bone strength.

Currently approved drugs for treatment of osteoporosis in Europe, namely bisphosphonates, denosumab, and teriparatide all decrease vertebral fracture risk within one year, whereas the benefits on non-vertebral fractures may take nearly two years to appear.

Objectives: To provide an update of recent trials regarding osteoporosis treatment.

Methods: Literature review and expert insights into recent analyses of clinical trials.

Results: $\mathrm{New} \mathrm{H} 2 \mathrm{H}$ trials, notably with teriparatide compared to risedronate, have shown the superiority of the anabolic therapy in preventing fractures in high risk patients. In GIOP, denosumab increased BMD more than risedronate. More importantly, romosozumab, a monoclonal Ab against sclerostin, was superior to alendronate to reduce fractures in high risk subjects, whereas the sequence of romosozumab and denosumab was superior to placebo followed by denosumab and increased BMD over just two years equivalent to seven years of continuous denosumab. New analyses of long-term denosumab, up to ten years of continuous therapy, have shown continuous BMD gains and further reduction in fracture rates, with T-scores at hip near -1.5 achieving the lowest fracture risk. However several case reports as well as a post-hoc analysis of subjects discontinuing denosumab in the FREEDOM trial have reported an increased risk of multiple vertebral fractures, which may be prevented by a 1-2 yrs consolidation by a bisphosphonate following denosumab therapy (guidelines).

Conclusion: In summary, while new data and more potent drugs are emerging (romosozumab), fueling the concept that anabolics should be used earlier in the course of therapy in high risk patients, additional long-term data with anti-resorptives indicate that continuous treatment, at least as long as treatment targets ( $T$ scores $>-2$ and no fractures) are not reached, has a favourable benefits/risk profile.

Disclosure of interests: None declared

DOI: 10.1136/annrheumdis-2019-eular.8475

WEDNESDAY, 12 JUNE 2019

14:15:00 - 15:45:00

\section{Best practices in spondyloarthritis}

\section{SP0003 NSAIDS - HOW TO OPTIMALLY USE THEM? (SAFETY OF NSAIDS)}

Victoria Navarro-Compán. University Hospital La Paz, IdiPaz, Rheumatology, Madrid, Spain

The management of patients with axial spondyloarthritis (axSpA) requires a combination of non-pharmacological and pharmacological treatment modalities.
Among pharmacological treatment, nonsteroidal anti-inflammatory drugs (NSAIDs) are recommended as first-line drug. NSAIDs have shown to be efficacious to reduce symptoms and signs in patients with axSpA.

Nevertheless, NSAIDs are not exepmt of side effects especially when adminis tered chronically, as in patients with axSpA. These include gastrointestinal, cardiovascular and nephrological side effects. In addition, some situations may influence on the prescription of NSAIDs, such as the presence of some extrarheumatolgical manifestations of the disease (inflammatory bowel disease), comorbidities and family planning-decisions including pregnancy and breastfeeding. Therefore, it is essential taking risk and benefits of NSAIDs use to properly manage patients with axSpA. During this presentation, the optimal usage of NSAIDs in patients with axSpA will be analysed and discussed, taken into account all the possible side effects and the context of the patient.

Disclosure of Interests None declared

DOI: 10.1136/annrheumdis-2019-eular.8547

\section{SP0004 MANAGING PATIENTS WITH AXSPA: WHAT ARE THE QUALITY STANDARDS?}

Uta Kiltz. Rheumazentrum Ruhrgebiet at Ruhr-University Bochum, Rheumatology, Herne, Germany

Background: There is wide variation in the management of patients with axial spondyloarthritis (axSpA) worldwide with significant unmet needs such as delayed diagnosis and inequity in bMARD prescription. ${ }^{1}$ Assessing the quality of care provided to patients with axSpA is important not only to patients and physicians, but also to providers and purchasers of health care. There is no agreed methodology to quantify quality of care but several approaches have been proposed aiming to assess quality in a measurable construct, e.g. quality indicators, performance measures or quality standards. Definition of quality standards (QS) enable society to identify resources and processes which may need to be optimized in patients with axSpA.

Objectives: A major goal of the international organization Assessment of SpondyloArthritis international Society (ASAS) is to improve quality of care and health outcomes in patients with axSpA. Recognized gaps in current care prompted ASAS in 2016 to start developing a quality standard set (ASAS QS) to optimize access, treatment and patient outcomes in axSpA.

Methods: An ASAS task force developed a set of ASAS QS step-wise. First, key areas for quality improvement were proposed, discussed, rated and agreed on Thereafter, key areas were prioritized and statements for the most important key areas were phrased on consensus. Appropriate tools were selected and measures developed to be able to assess and quantify the quality of care on the community level.

Results: The ASAS task force, consisting of 20 rheumatologists, 2 physiotherapists and 2 patients, selected and proposed 34 potential key areas for quality improvement which were commented by 140 ASAS survey participants (86 physicians, 42 patients). Within that process 3 new key areas came up, which led to a reevaluation of all 37 key areas by 120 participants ( 86 physicians, 29 patients). Five key areas were identified as most important to determine quality of care: referral including rapid access, monitoring, treatment, education including patient information and comorbidities. On that background, 9 QS were agreed on and finally endorsed by ASAS.

Conclusion: ASAS successfully developed the first QS set for improvement of health care for adult patients with axSpA. All QS are measurable achievable in daily care in an optimized situation and intend to minimize variation in quality of care.

\section{REFERENCE:}

[1] Nikiphorou E, et al. Inequity in biological DMARD prescription for spondyloarthritis across the globe: results from the ASAS-COMOSPA study. Ann Rheum Dis 2018;77:405-411.

Disclosure of interests: Uta Kiltz Grant/research support from: AbbVie, Chugai, Eli Lilly, Grünenthal, Janssen, MSD, Novartis, Pfizer, Roche, and UCB., Consultant for: AbbVie, Chugai, Eli Lilly, Grünenthal, Janssen, MSD, Novartis, Pfizer, Roche, and UCB. DOI: 10.1136/annrheumdis-2019-eular.8514 
WEDNESDAY, 12 JUNE 2019

14:15:00 - 15:45:00

\section{How to maintain remission in vasculitis}

\section{SP0005 CASE 1 DISCUSSANT: LONG TERM EFFICACY OF REMISSION-MAINTENANCE REGIMENS FOR AAV}

Loïc Guillevin. National Referral Center for Rare Systemic and Autoimmune Diseases, Hôpital Cochin, Assistance Publique-Hôpitaux de Paris, Université Paris Descartes, Department of Internal Medicine, Paris, France

ANCA-associated vasculitides (AAVs) are a group of diseases with frequent relapses that can sometimes be severe. Treatment comprises an induction-remission phase followed by a maintenance regimen. Induction-remission therapy is effective and now well-established, mainly based on the combination of corticosteroids (CS) and cyclophosphamide or rituximab. For decades, maintenance therapy was compulsory and consisted of azathioprine or methotrexate, combined or not with low-dose CS. That regimen was prescribed for at least 18 months but was sometimes taken for several years. Criteria for stopping treatment have never been codified. Since rituximab has been widely prescribed for induction, the indication for azathioprine maintenance has been challenged because its relapse rate at 18 months $(29 \%)$ was comparable to that of placebo (32\%) given in its stead. However, we considered those relapse rates unacceptably high and have tried to devise a different therapeutic approach to maintenance, with semestrial evaluation of the indication of rituximab infusion over 18 months. MAINRITSAN1trial results demonstrated the superiority of rituximab to maintain remission with a $5 \%$ relapse rate at 28 months vs $29 \%$ for azathioprine recipients. The 60 -month follow-up of that trial confirmed the superiority of rituximab over azathioprine $(37 \%$ vs $57 \%$ ), even though rituximab did not abrogate relapses. Since the publication of those results, it is now clear that AAV remission-maintenance therapy should be rituximab - and not azathioprine or another equally effective immunosuppressant like methotrexate.

Maintaining remission should now also be based on other factors predictive of relapse: vasculitis type, ANCA subtype and/or ANCA presence or absence at the end of the induction regimen. Granulomatosis with polyangiitis relapses more frequently than microscopic polyangiitis or eosinophilic granulomatosis with polyangiitis. Relapse rates of the former two are probably linked mainly to ANCA type, anti-PR3 or anti-MPO, with the latter relapsing less frequently. The long-term MAINRITSAN1-trial results identified anti-PR3 presence 1 year after ending induction treatment and/or their persistence were predictor(s) of relapse.

We also designed a study comparing fixed-schedule rituximab infusions to re-infusions[JJ1] guided by ANCA titer and/or CD19+ circulating B lymphocytes. Results of that prospective study demonstrated that fewer rituximab infusions should be given but that ANCA titer and the circulating CD19+ B-cell level are not good predictors of relapse. ANCA presence - independently of their titer-is more frequently associated with relapse.

The optimal rituximab-administration duration has not yet been established. Our group is now awaiting the imminent results of a prospective trial comparing 4 vs 8 rituximab infusions at 6-month intervals after obtaining remission.

Conclusions: Major advances have been made in the therapeutic strategy for AAVs. After remission is obtained: 1) AAVs require maintenance therapy, 2) rituximab is superior to immunosuppressants, 3) the presence, persistence and/or reoccurrence of anti-PR3 ANCA predict relapses, 4) the 500-mg rituximab dose/ infusion seems well-adapted. The optimal treatment duration remains to be elucidated.

Tailoring maintenance therapy is now the main therapeutic objective for AAV management. Future trials will attempt to evaluate very long-term maintenance treatment of AAVs with the goal of eradicating relapses.

Disclosure of Interests: None declared

DOI: 10.1136/annrheumdis-2019-eular.8489

\section{SP0006 CASE 2 DISCUSSANT: LONG TERM EFFICACY OF REMISSION-MAINTENANCE REGIMENS FOR GPA}

Peter Villiger. inselspital, Rheumatology, Immunology and Allergology, Bern, Switzerland

Background: A case of Giant Cell Arteritis with different visual symptoms and delayed diagnosis will be presented. This 74 year-old patient was pulsed with Glucocorticoids (GC) and thereafter treated with Tocilizumab (TCZ). She rapidly achieved full and lasting remission. After 12 months of treatment with TCZ, infusions were stopped. The patient suffered minor relapse 4 months after treatment discontinuation and again responded to TCZ. The case raises several questions such as: How to dose and how long to treat with TCZ? How to reduce dose and when to stop GC? Are there variables to quantify subclinical diseases activity as CRP and ESR are unreliable under TCZ treatment? Are there predictive factors for relapse after treatment discontinuation? How to control development of disease damage over time?

Disclosure of Interests: None declared

DOI: 10.1136/annrheumdis-2019-eular.8615

WEDNESDAY, 12 JUNE 2019

14:15:00 - 15:45:00

\section{CPPD - a forgotten disease that requires more attention??!!!}

\section{SP0007 IS ULTRASOUND A GOOD TOOL TO EVALUATE CPPD?}

Georgios Filippou. University of Ferrara, Rheumatology Department, Ferrara, Italy

CPPD is an arthropathy with high prevalence among the elderly and a multitude of clinical manifestations that can easily bring to misdiagnosis and therapeutic errors. The possibility to assess different aspects of the disease with only one too can be of great help for clinicians.

Ultrasound (US) can help to address both the diagnosis and the inflammatory assessment of the disease. In the last years a lot of research has been carried out regarding the applications of US in CPPD. The special OMERACT US sub-task force that is working in CPPD has produced new definitions for identification of CPPD by US and has tested their reliability demonstrating that US can be used reliably at the knees and wrists for assessing CPPD[1,2]. Further, US has been tested also for assessment of inflammatory and structural changes in osteoarthritis demonstrating to be reliable also for this purpose[3].

During the lecture the potential of US in diagnosis and follow-up of patients with CPPD will be presented. Further, a deeper insight into possible pathogenetic mechanisms, based on observations made at US imaging, will be discussed.

\section{REFERENCES}

[1] Filippou G., Scirè C.A., Damjanov N., et al. Definition and reliability assessment of elementary ultrasonographic findings in calcium pyrophosphate deposition disease: A study by the OMERACT calcium pyrophosphate deposition disease ultrasound subtask force. J Rheumato 2017;44:1744-9. doi:10.3899/jrheum.161057

[2] Filippou G, Scirè CA, Adinolfi A, et al. Identification of calcium pyrophosphate deposition disease (CPPD) by ultrasound: reliability of the OMER ACT definitions in an extended set of joints - an international multiobserver study by the OMERACT Calcium Pyrophosphate Deposition Disease Ultrasound Subtask Force. Ann Rheum Dis 2018::annrheumdis-2017-212542. doi:10.1136/annrheumdis-2017-212542

[3] Bruyn GA, Naredo E, Damjanov N, et al. An OMERACT reliability exercise of inflammatory and structural abnormalities in patients with knee osteoarthritis using ultrasound assessment. Ann Rheum Dis Published Online First: 22 April 2015. doi:10.1136/annrheumdis-2014-206774

Disclosure of Interests: Georgios Filippou Speakers bureau: Laborest, Abbvie, BMS, Sanofi

DOI: 10.1136/annrheumdis-2019-eular.8480

\section{SP0008 MANAGEMENT OF CPPD DISEASE}

Abhishek Abhishek. The University of Nottingham, Academic Rheumatology, Nottingham, United Kingdom

Background: Calcium pyrophosphate deposition (CPPD) is a common cause of arthritis. Its' prevalence increases with ageing, and it manifests with asymptomatic chondrocalcinosis, acute crystal synovitis, and chronic arthritis.

Objectives: The objectives of this talk are to summarize the treatment options for the management of CPPD, and, to review the evidence base supporting them. Methods: A systematic literature search was performed to identify all studies published in the English language, and, reporting on the treatment of acute and chronic manifestations of CPPD. All published studies were included with the exception of case reports and conference abstracts. Similarly, a literature search was performed to identify the metabolic and hereditary risk-factors of CPPD. The findings of the systematic literature search are described in a narrative manner. Interventions for which there is no published data are recommended based on clinical experience and expert opinion.

Results: Based on clinical experience, oral or intra-articular corticosteroids are recommended for the management of CPPD. Colchicine and interleukin-1 antag onists are effective and recommended for the management of acute CPP crystal arthritis. Interleukin-1 antagonists should be reserved for use in refractory cases. Oral NSAIDs should be avoided as people with CPPD are frequently elderly. Low- 